\title{
Results on Proton-Irradiated 3D Pixel Sensors Interconnected to RD53A Readout ASIC
}

\author{
J. Duarte-Campderros ${ }^{\mathrm{a}, \mathrm{b}, *}$, on behalf of the CMS Collaboration,

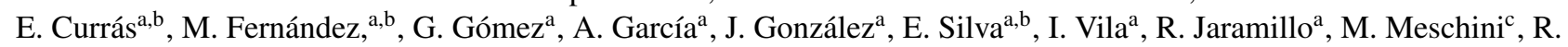

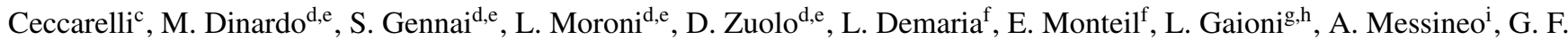

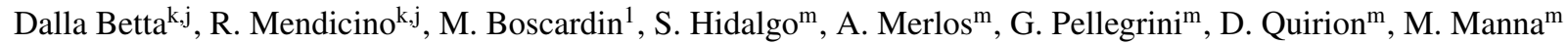 \\ ${ }^{a}$ Instituto de Fisica de Cantabria (Universidad de Cantabria/CSIC), Av. de los Castros s/n 39005 Santander, Spain \\ ${ }^{b}$ European Organization for Nuclear Research (CERN), 1211 Geneva 23, Switzerland \\ ${ }^{c}$ INFN Sezione de Firenze, Via Giovanni Sansone, 1, 50019 Sesto Fiorentino FI, Italy \\ ${ }^{d}$ INFN Sezione di Milano-Bicocca, Milano, Italy \\ ${ }^{e}$ University of Milano Bicocca, Milano, Italy

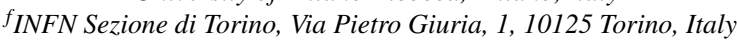 \\ ${ }^{g}$ INFN Sezione di Pavia, Via Agostino Bassi, 6, 27100 Pavia, Italy \\ ${ }^{h}$ Universita degli studi di Bergamo, Bergamo, Italy \\ ${ }^{i}$ INFN Sezione di Pisa and Universita di Pisa, Largo Bruno Fibonacci, 2, 56127, Pisa, Italy \\ ${ }^{j}$ Università degli Studi di Trento, Via Calepina, 14, 38122 Trento, Italy \\ ${ }^{k}$ INFN Sezione di Padova, Gruppo Collegato di Trento, Trento, Italy \\ ${ }^{l}$ FBK-Fondazione Bruno Kessler, Via Sommarive, 18 - Povo 38123 Trento, Italy \\ ${ }^{m}$ Centro Nacional de Microelectrónica, IMB-CNM (CSIC), 08193 Barcelona, Spain
}

\section{Abstract}

Test beam results obtained with 3D pixel sensors bump-bonded to the RD53A prototype readout ASIC are reported. Sensors from FBK (Italy) and IMB-CNM (Spain) have been tested before and after proton-irradiation to an equivalent fluence of about $1 \times 10^{16} \mathrm{n}_{\mathrm{eq}} \mathrm{cm}^{-2}$ ( $1 \mathrm{MeV}$ equivalent neutrons). This is the first time that one single collecting electrode fine pitch 3D sensors are irradiated up to such fluence bump-bonded to a fine pitch ASIC. The preliminary analysis of the collected data shows no degradation on the hit detection efficiencies of the tested sensors after high energy proton irradiation, demonstrating the excellent radiation tolerance of the 3D pixel sensors. Thus, they will be excellent candidates for the extreme radiation environment at the innermost layers of the HL-LHC experiments.

Keywords: Performance of High Energy Physics Detectors, Pixelated detectors and associated VLSI electronics, Radiation-hard electronics, Radiation damage to detector materials (solid state), Radiation-hard detectors

\section{Radiation Hardness and 3D Columnar Pixel Sensors}

Pixel detectors in the innermost layers of the High Luminosity Large Hadron Collider (HL-LHC) experiments will have to survive a fluence in excess of $1 \times 10^{16} \mathrm{n}_{\mathrm{eq}} \mathrm{cm}^{-2}$ ( $1 \mathrm{MeV}$ equivalent neutrons), while preserving high tracking efficiency [1]. It has already been demonstrated [2] that 3D pixel sensors are sufficiently radiation tolerant to resist the expected fluence on the innermost layers of the tracking systems of HL-LHC experiments, although it was done by using a coarse pitch readout chip. This work, complementing ref. [3], uses for the first time 3D sensors bump-bonded to a fine pitch readout ASIC (RD53A). In addition, the study presents characterization results of 3D pixel sensors of $25 \mu \mathrm{m} \times 100 \mu \mathrm{m}$ pitch with one single collecting electrode per cell, irradiated up to $1 \times 10^{16} \mathrm{n}_{\mathrm{eq}} \mathrm{cm}^{-2}$.

Columnar 3D pixel sensors [4, 5] possess several intrinsic properties making them good candidates to create the layers of

\footnotetext{
${ }^{*}$ Corresponding author. Tel.: +41 227671657

Email address: jorge.duarte.campderros@cern.ch (J. Duarte-Campderros)
}

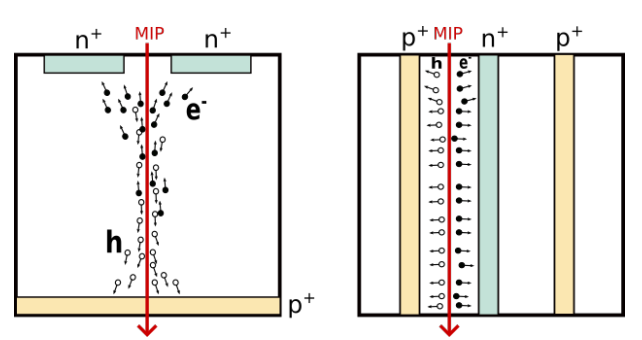

Figure 1: Schematic diagram of a planar (left) and a 3D (right) sensor, showing the charge carrier creation when a particle passes through, and the carriers movement before being collected on the electrodes.

a tracking system exposed to an extreme radiation environment. For example, the voltage needed to fully deplete the sensor is much lower than in an equivalent planar sensor, particularly after irradiation, due to small distance between junction and ohmic contacts. For the same reason, charge carriers travel less distance than in an equivalent planar detector, therefore they are less impacted by trapping. Charge carriers in the 3D pixel sensors travel only $35 \mu \mathrm{m}$ for a $50 \mu \mathrm{m} \times 50 \mu \mathrm{m}$ pixel pitch independently of the sensor thickness, which is the driving parameter 
for planar pixels. The 3D pixels have also a very fast response time. Figure 1 compares both planar and 3D designs.

The 3D sensors used in this work were fabricated at the FBK in Trento (Italy), and in the IMB-CNM in Barcelona (Spain). Sensors from FBK were bump-bonded to the RD53A [6] readout-chip, while sensors from IMB-CNM were bumpbonded to the ROC4SENS [7] chip. The substrates were p-type Si-Si high-resistivity wafers with $130 \mu \mathrm{m}$ thickness in the case of the FBK sensors, and conventional p-type high-resistivity FZ wafers of $230 \mu \mathrm{m}$ thickness, for the IMB-CNM sensors. Detector and fabrication description can be found on ref. [4, 5]. Figure 2 shows both single-sided FBK and double-sided IMBCNM pixel layout sensors.

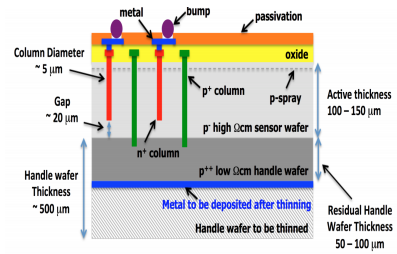

(a) Single-sided 3D FBK

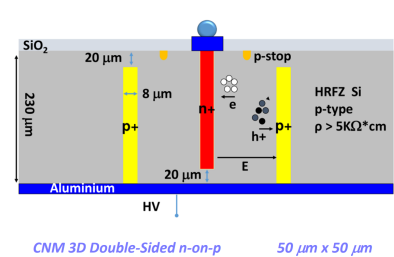

(b) Double-sided 3D CNM
Figure 2: Cross-section of the 3D pixel sensor layouts

The sensors were created with two different pixel cells: $50 \mu \mathrm{m} \times 50 \mu \mathrm{m}$ and $25 \mu \mathrm{m} \times 100 \mu \mathrm{m}$, the latter having one (1E) or two (2E) collecting electrodes per cell. The $1 \mathrm{E}$ case is particularly interesting given the large yield when producing them. Two examples of pixel cells are shown in figure 3 Both cell sizes are presently under evaluation in the Compact Muon Solenoid (CMS) collaboration for the inner layers of the pixel detectors for HL-LHC.

After fabrication, the FBK pixel sensor wafers were processed for UBM (Under Bump Metallization), thinned down to $200 \mu \mathrm{m}$ total thickness, diced and bump-bonded to RD53A prototype chips at IZM (Berlin, Germany). The RD53A chip has 76800 readout channels (400 rows and 192 columns with a bump pad pitch of $50 \mu \mathrm{m} \times 50 \mu \mathrm{m})$ and measures $20.0 \mathrm{~mm} \times 11.8 \mathrm{~mm}$. The pixel sensor bonded to the readout chip needs to be glued and wire-bonded onto an adapter card in order to be tested; these units will be referred to as modules in the following text. All results referring to the FBK+RD53A modules were obtained with the Linear Front-End [? ] (FE) in the central zone of RD53A (136 columns wide, from 128 to 263).

IMB-CNM sensors were also processed for UBM and then bump-bonded to ROC4SENS readout chips. The ROC4SENS is a generic chip able to readout without zero suppression, and therefore specially well suited for sensor studies. The chip has a bump pad pitch of $50 \mu \mathrm{m} \times 50 \mu \mathrm{m}$ with 160 rows and 155 columns providing 24800 pixels in a $9.8 \mathrm{~mm} \times 7.8 \mathrm{~mm}$ surface.

\section{Irradiation and Test Beam setup}

Irradiations were performed in 2017 for the IMB-CNM sensors, and in 2018 for the FBK sensors at the CERN IRRAD

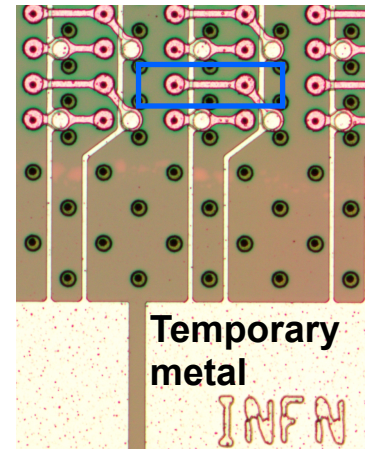

(a) $25 \times 100 \mu \mathrm{m}^{2} 2 \mathrm{E}$ sensor

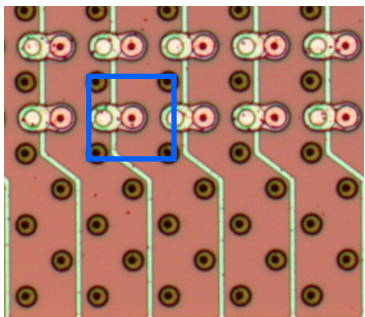

Temporary metal

inजाए की

(b) $50 \times 50 \mu \mathrm{m}^{2}$ sensor
Figure 3: Microscope pictures of 3D FBK sensors. The contact pad for the probe and the vertical metal lines connecting all pixels are visible in the pictures by the Temporary metal label, used for sensor testing at FBK premises and which is subsequently removed. A $3 \mathrm{D}$ single pixel cell on each sensor is highlighted with the blue frames. Note the metal routing configuration in (a) in order to connect the readout electrodes to the square matrix RD53A bumping pads.

facility [8], a high intensity $24 \mathrm{GeV} / \mathrm{c}$ proton beam which has a FWHM of $12 \mathrm{~mm}$ in $\mathrm{x}$ and $\mathrm{y}$ directions. The target fluence for the IMB-CNM sensors was $3 \times 10^{15} \mathrm{n}_{\mathrm{eq}} \mathrm{cm}^{-2}$, and $1 \times 10^{16} \mathrm{n}_{\mathrm{eq}} \mathrm{cm}^{-2}$ for the FBK ones. The latter modules were tilted on the IRRAD beam at an angle of $55^{\circ}$ in order to homogeneously irradiate the $20 \mathrm{~mm} \times 12 \mathrm{~mm}$ sensors and readout chip areas. The corresponding total ionizing dose was $6 \mathrm{MGy}$ for $1.65 \times 10^{16}$ protons $\mathrm{cm}^{-2}$.

Visual inspections after irradiation and data analysis are showing that the FBK modules were displaced with respect to the irradiation beam axis by a few millimeters and therefore the nominal fluence was reached only in a part of the modules, in particular in about half of the linear FE. Several measurements and cross-checks are being performed in order to establish the effective fluence integrated on the modules. All results shown here are based on the nominal requested equivalent fluence. Figure 4 shows several modules mounted on the supports ready to be irradiated and one of the modules characterized in this work.

RD53A modules were tested in two test beam experiments at the CERN North Area H6B before and after irradiation in July and October 2018 respectively. The test beam facility at CERN provided for this study $120 \mathrm{GeV} / \mathrm{c}$ hadrons, allowing to characterize the sensor response to minimum ionizing particles. Particle trajectories are measured by the high-spatial-resolution planes of an EUDET-type telescope [9] with less than $5 \mu \mathrm{m}$ accuracy, and spatially correlated with the hits measured at our modules, i.e. Devices Under Test (DUT). An extra plane is placed between the telescope planes and is used to correlate in time the telescope with the DUT hits. The hit efficiency of a DUT is calculated from the available trajectories with a hit in the reference sensor, and looking for a hit in the DUT. More details on an equivalent test beam setup and data analysis can be found at ref. [10].

Irradiated modules were kept cold at temperatures between $-20^{\circ} \mathrm{C}$ and $-30^{\circ} \mathrm{C}$ using dry ice bricks. The temperature was 


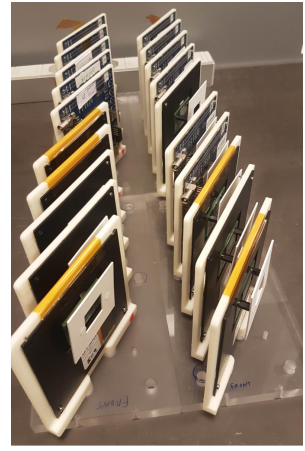

(a) Modules mounted on the tilted supports

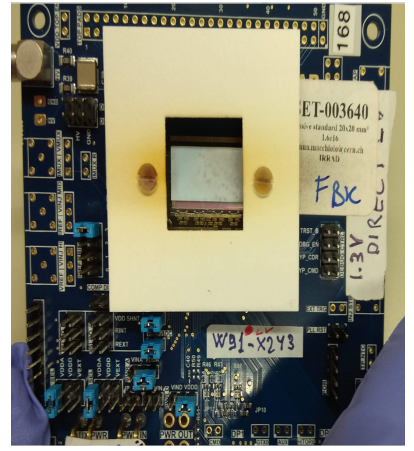

(b) A module after irradiation
Figure 4: Tilted modules mounted on the irradiation tray (a). A module after irradiation (b), the dark brown band on the cardboard frame and on the nylon screw heads is due to the proton beam passing through the tilted module.

monitored via PT1000 sensors located close to the backside of the module and via NTC resistors soldered on the adapter card. Both sensors gave consistent measurements.

The readout chip parameters were tuned in order to reach low thresholds and noise, having at most $1.5 \%$ masked pixels because of noisy channels. For the irradiated modules the average signal threshold was set to about 1400 electrons, with a noise value of 105 electrons for non-masked pixels, as shown in figure 5 for a $25 \mu \mathrm{m} \times 100 \mu \mathrm{m}$. Similar parameters were found for the $50 \mu \mathrm{m} \times 50 \mu \mathrm{m}$. The color scale for the threshold distri-

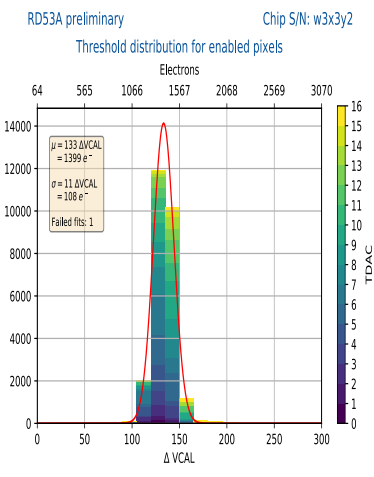

(a) Module Threshold

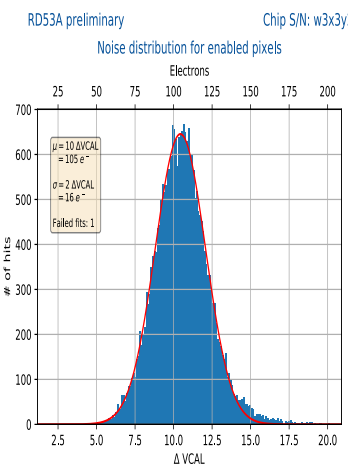

(b) Module noise
Figure 5: Signal threshold (a) and noise (b) distributions for a $25 \mu \mathrm{m} \times 100 \mu \mathrm{m}$ module after irradiation.

bution represents the 4-bit DAC value used for the trimming of each individual pixel response.

\section{Sensor response and Results}

The 3D modules prior to irradiation reached hit detection efficiencies above $98.5 \%$ for perpendicular incident tracks, already at moderate bias (less than $15 \mathrm{~V}$ ).

After irradiation, a bias voltage of at least $120 \mathrm{~V}$ was needed to reach similar efficiency. Figure 6 shows the comparison of hit
Table 1: Hit detection efficiency summary table for a fluence of $1 \times 10^{16} \mathrm{n}_{\mathrm{eq}} \mathrm{cm}^{-2}$ (errors are not quoted).

\begin{tabular}{lcc}
\hline 3D Pixel-RD53A Linear FE & $25 \mu \mathrm{m} \times 100 \mu \mathrm{m}$ & $50 \mu \mathrm{m} \times 50 \mu \mathrm{m}$ \\
\hline \hline Before irradiation & $97.3 \%$ & $98.6 \%$ \\
After irradiation & $96.6 \%$ & $97.5 \%$ \\
\hline
\end{tabular}

efficiency before and after irradiation for $25 \mu \mathrm{m} \times 100 \mu \mathrm{m}$, and figure 7 for $50 \mu \mathrm{m} \times 50 \mu \mathrm{m}$ pixel size modules, and perpendicular incident tracks. In the efficiency plots the hits reconstructed over the whole module are projected on a $2 \times 2$ pixel cell window to put in evidence the sensor geometry and the possible effects of the columnar electrodes. The geometrical inefficiency

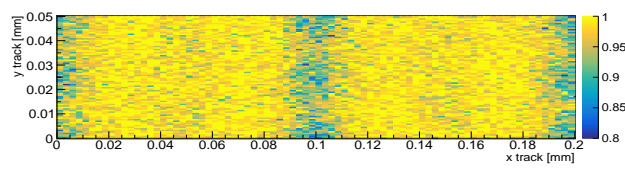

(a) Efficiency before irradiation at $3 \mathrm{~V}$ bias

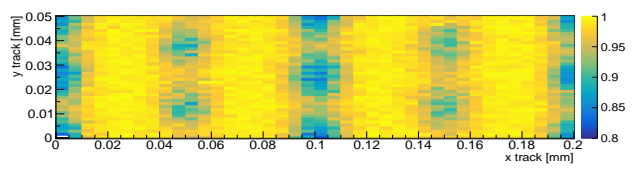

(b) Efficiency after irradiation at $120 \mathrm{~V}$ bias

Figure 6: Hit detection efficiencies before (a) and after irradiation (b) for a $25 \mu \mathrm{m} \times 100 \mu \mathrm{m}$ module.

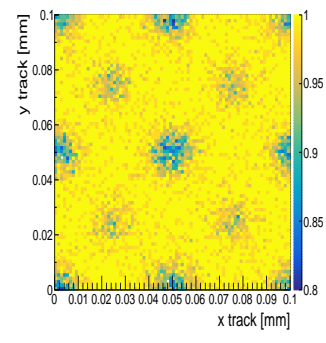

(a) Efficiency before irradiation at (b) Efficiency after irradiation at $15 \mathrm{~V}$ bias

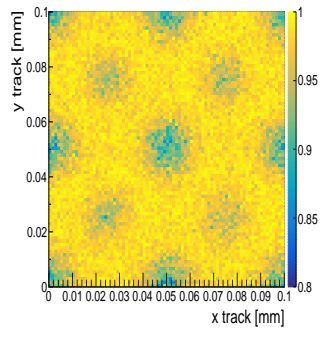

$150 \mathrm{~V}$ bias
Figure 7: Hit detection efficiencies before (a) and after irradiation (b) for a $50 \times 50 \mu \mathrm{m}^{2}$ module.

due to the columnar electrode diameter of $5 \mu \mathrm{m}$ was estimated to be around $1.5 \%$. This effect can be greatly reduced by tilting the module on the beam. Hit efficiency higher than $99.3 \%$ was recovered when sensors are tilted with respect to the incident particles in order to maximize the charge sharing between pixels, as shown in figure 8 This proves that the tested 3D sensors, both $25 \mu \mathrm{m} \times 100 \mu \mathrm{m}$ and $50 \mu \mathrm{m} \times 50 \mu \mathrm{m}$ are radiation tolerant up to the extreme expected doses at the HL-LHC. In particular, in the case of the $25 \mu \mathrm{m} \times 100 \mu \mathrm{m}, 1$ electrode is enough to reach high hit efficiency. ROC4SENS modules were tested at the DESY test beam facility [11] with $5 \mathrm{GeV} / \mathrm{c}$ electrons. 
As expected no significant reduction of the sensor hit efficiency was observed after irradiation and overall efficiency of $98 \%$ at normal incidence was reached.

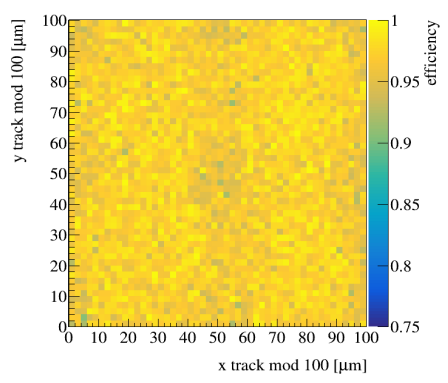

Figure 8: Hit detection efficiencies when the sensor planes were tilted with respect to the incident particles by $12^{\circ}$. This figure corresponds to a $50 \mu \mathrm{m} \times 50 \mu \mathrm{m}$ IMB-CNM sensor irradiated with a fluence of $3 \times 10^{15} \mathrm{n}_{\mathrm{eq}} \mathrm{cm}^{-2}$, and bump-bonded to a ROC4SENS. The bias voltage was $150 \mathrm{~V}$.

The hit detection efficiencies as calculated in our data analysis for different runs are reported in table 1 .

The hit efficiency as a function of the applied bias voltage is reported in figure 9, where it can be observed that starting from $120 \mathrm{~V}$ the sensors reach the full depletion regime.

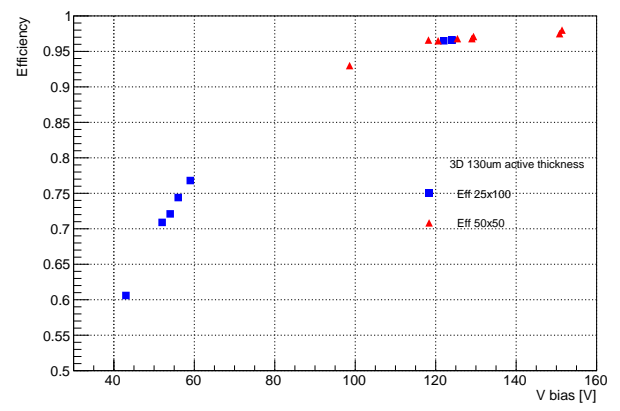

Figure 9: Hit detection efficiency as a function of the bias voltage for irradiated sensors up to a fluence of $1 \times 10^{16} \mathrm{n}_{\mathrm{eq}} \mathrm{cm}$; for $50 \mu \mathrm{m} \times 50 \mu \mathrm{m}$ (red triangles) and $25 \mu \mathrm{m} \times 100 \mu \mathrm{m} 1 \mathrm{E}$ (blue squares), with perpendicular incident particles.

\section{Conclusions and Outlook}

Initial test beam results obtained with 3D pixel sensors show no significant degradation after proton irradiation up to $1 \times 10^{16} \mathrm{n}_{\mathrm{eq}} \mathrm{cm}$ at bias voltages below $200 \mathrm{~V}$, confirming 3D pixel sensors as a possible robust option for the inner layers of future tracking detectors. In particular, it is shown that one single collecting electrode per cell is enough to assure high efficiency. New data is currently being analyzed from the CERN 2018 test beam campaign as well as from Fermilab and DESY test beams, in order to confirm and extend this study. At the beginning of 2019, a new 3D sensor batch was in production at FBK and a new batch with $25 \mu \mathrm{m} \times 100 \mu \mathrm{m}$ and $50 \mu \mathrm{m} \times 50 \mu \mathrm{m}$ pitch sensors just finished at IMB-CNM. These will be tested and characterized throughout 2019.

\section{Acknowledgments}

I wish to thank Nuria Castello-Mor for providing me the elegant template I used for the poster. We thank the RD53 Collaboration for the RD53A chip; we remind our results are not on chip performance but on sensor performance. The RD50 Collaboration for its support. Bonn ATLAS group for SCC cards and support for flip-chipping. Some of the measurements leading to these results have been performed at the CERN North Area Test Beam Facility at Prévessin (France). Some of the measurements leading to these results have been performed at the Test Beam Facility at DESY Hamburg (Germany), a member of the Helmholtz Association (HGF). This project has been partially supported by the Spanish Ministry of Science under grants FPA2015-71292-C2-2-P, FPA2017-85155-C4-1-R and FPA2017-85155-C4-2-R; and the European Union's Horizon 2020 Research and Innovation programme under Grant Agreement no. 654168 (AIDA-2020).

\section{References}

[1] CMS Collaboration, The Phase-2 Upgrade of the CMS Tracker Tech. Rep. CERN-LHCC-2017-009. CMS-TDR-014, CERN, Geneva (Jun 2017).

URL https://cds.cern. ch/record/2272264

[2] J. Lange, G. Giannini, S. Grinstein, M. Manna, G. Pellegrini, D. Quirion, S. Terzo, D. Vzquez Furelos, Radiation hardness of small-pitch 3D pixel sensors up to a fluence of $3 \times 10^{16} \mathrm{n}_{\mathrm{eq}} / \mathrm{cm}^{2}$, JINST 13 (09) (2018) P09009. arXiv:1805.10208 doi:10.1088/1748-0221/13/09/P09009

[3] M. Meschini, et al., First Results on 3D Pixel Sensors Interconnected to the RD53A Readout Chip after Irradiation to $1 \times 10^{16}$ neq $\mathrm{cm}^{-2}$, in: 9 th International Workshop on Semiconductor Pixel Detectors for Particles and Imaging (PIXEL 2018) Taipei, Taiwan, December 10-14, 2018, 2019. arXiv: 1903.01963

[4] G.-F. Dalla Betta, R. Mendicino, D. Sultan, M. Boscardin, G. Giacomini, S. Ronchin, N. Zorzi, G. Darbo, M. Meschini, A. Messineo, Small pitch 3D devices, PoS Vertex2016 (2017) 028. doi:10.22323/1.287.0028

[5] G. Pellegrini, J. P. Balbuena, D. Bassignana, E. Cabruja, C. Fleta, C. Guardiola, M. Lozano, D. Quirion, M. Ullan, 3D double sided detector fabrication at IMB-CNM, Nucl. Instrum. Meth. A699 (2013) 27-30. doi:10.1016/j.nima.2012.05.087

[6] M. Garcia-Sciveres, The RD53A Integrated Circuit Tech. Rep. CERNRD53-PUB-17-001, CERN, Geneva (Oct 2017).

URL https : //cds.cern.ch/record/2287593

[7] R. Horisberger, H.-C. Kaestli, B. Meier, R. Tilman, S. Wiederkehr, ROC4SENS - a generic readout chip for sensor studies, https: //indico.cern.ch/event/663851/contributions/2788211. Accessed: 2019-03-01.

[8] PS-IRRAD Proton Facility, https://ps-irrad.web.cern.ch accessed: 2019-02-12.

[9] H. Jansen, S. Spannagel, A. Bulgheroni, G. Claus, E. Corrin, D. Cussans, J. Dreyling-Eschweiler, D. Eckstein, T. Eichhorn, M. Goffe, I. Gregor, D. Haas, C. Muhl, H. Perrey, R. Peschke, P. Roloff, I. Rubinskiy, M. Winter, Performance of the EUDET-type beam telescopes EPJ Tech. Instrum. 3 (arXiv:1603.09669. DESY 16-055) (2016) 7. 18 p. URL http: //cds . cern. ch/record/2143018

[10] E. Currs, J. Duarte-Campderrs, M. Fernndez, A. Garca, G. Gmez, J. Gonzlez, R. Jaramillo, D. Moya, I. Vila, S. Hidalgo, M. Manna, G. Pellegrini, D. Quirion, D. Pitzl, A. Ebrahimi, T. Rohe, S. Wiederkehr, First study of small-cell 3D Silicon Pixel Detectors for the High Luminosity LHC NIM A (arXiv:1806.01435) (2018) 20 p, 20 pages, 19 figures.

URL https : //cds.cern.ch/record/2622152

[11] R. Diener, et al., The DESY II Test Beam Facility, Nucl. Instrum. Meth. A922 (2019) 265-286. arXiv:1807.09328 doi:10.1016/j.nima. 2018.11.133 